\begin{tabular}{ccc}
\hline & International Journal of Engineering \& Technology, $7(1.5)(2018)$ 196-201 \\
SPC & International Journal of Engineering \& Technology \\
\hline
\end{tabular}

\title{
Performance metric analysis of modified LEACH routing protocol in wireless sensor network
}

\author{
Raghul Priyadarshi *, Hitesh Tripathi, Abhyuday Bhardwaj, Ankush Thakur \\ Department of Electronics \& Communication Engineering, National Institute of Technology Hamirpur (H.P), India \\ *Corresponding author E-mail: rahul.glorious91@gmail.com
}

\begin{abstract}
The lifetime of a network is reflected as one of the vital concerns in wireless sensor network because of a huge number of nodes and further its density and distribution. When the network size increases then routing protocol becomes one of the crucial issues that which routing protocol one should use so that network lifetime to be enhanced. Furthermore, sensor nodes must be alive to promise that network process must continue without any interruption or any loss of data in the network. In fact, there are a lot of clustering method is there to augment the network lifetime. But in this paper, presented routing protocol that is based on existing LEACH protocol called as K-mean clustering routing $(\mathrm{KLEACH})$. Simulation results show that efficiency of the network has been improved and network lifetime has been also enhanced compared to existing LEACH protocol. Network lifetime of KLEACH protocol is almost increased by $40 \%$ and energy consumption is also decrease by almost $38 \%$ which reflects the good agreement of KLEACH algorithm compared to existing algorithm.
\end{abstract}

Keywords: Wireless Sensor Network, Clustering, Energy conservation, Sensor Network Lifetime, Routing protocols, Energy efficiency, Cluster-based routing.

\section{Introduction}

Wireless Sensor Networks (WSNs) have recently appeared as one of the vital key for several applications. The WSNs are generally used in various military (army, navy) as well as in civil uses[1][2]. Because of unfriendly installment of WSNs, it is not possible to replace the batteries of thousands of nodes that are installed in particular region[3]. Keeping in mind that installation of node in remote environment is very difficult, so the network need to be work autonomously deprived of any human involvement. Further, sensor nodes identified severe constraints of resources like bandwidth, memory storage and energy limitation[4]. Subsequently, the design of current sensor nodes that have quite a lot of confines provokes the job of detecting and reporting to be a vast discussion about performance adeptness troubles. Hence, the performances of WSNs like network lifetime and energy consumption have been fascinating the attention of numerous academics and there are numerous approaches to create this type of performance further efficient[5][6]. One of the main methodologies is clustering technique[7]. Indeed, the act which splits the system into several sets of sensor nodes is called as Clustering. Generally, a main node in the cluster called as Cluster Head $(\mathrm{CH})$ allows the attachment of all other nodes that belong to the same group by a measure of distance. Inside each of these clusters, $\mathrm{CH}$ gathers the data from sensor nodes that are attached to it. All member nodes transfer the data to their $\mathrm{CH}$. $\mathrm{CH}$ accumulates data packet from all the attached sensor nodes and advancing it to the Sink or one can say Base Station (BS)[8]. Figure 1 signifies the network where all the attached nodes transfer data packet to their $\mathrm{CH}$ and further $\mathrm{CH}$ sends collected data packet to their BS[9].

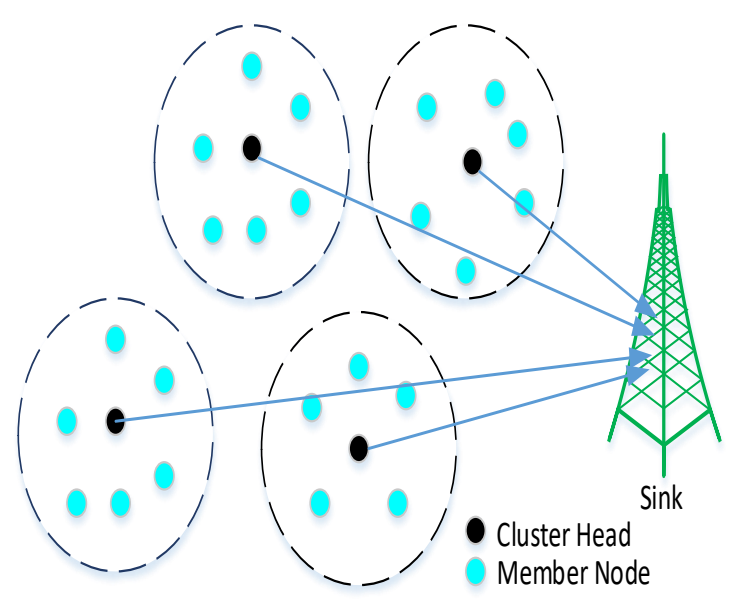

Fig.1: Clustering in WSN

Though, data packet routing is promising deprived of clusters, however necessity for a whole routing tabletop of every node is compulsory. Therefore, the utility of clustering is important to assure a well-organized routing[10]. Also, the nodes deployment and density have a significant effect on system scalability[11][12]. In reality, scalability is an important concern of a well-organized routing protocol. A noble routing protocol should scalable to any kind of change in the system topology and network size.

\section{Related works}

In [13][14]Author projected a routing protocol called as Low Energy Adaptive Clustering Hierarchy (LEACH) for similar or 
one can say homogeneous kind of WSN. In [15][16]Author selects node in a random way to form the $\mathrm{CH}$ and further distribute the energy uniformly among them. In [17][18]Author proposed LEACH protocol where they analyzed LEACH routing protocol in order of many factors like network lifetime, throughput and energy. In[19][20] author describe the LEACH protocol where $\mathrm{CH}$ selection is in randomized way but this further degrade the performance of LEACH protocol. In [21]authors proposed LEACH protocol where they were main focused on energy and throughput of the network. In [22] author examines the performance mainly for the sensor nodes energy and over-all network lifetime. Authors suggested an energy effective protocol on the basis of self-adaptive assembling of $\mathrm{CH}$. In this work we are going to propose a modified LEACH Protocol called as KLEACH protocol which enhances performance metric in WSN like number of data packets received, average data packets received, network lifetime and average energy consumption compared to all existing method.

\section{Performance metric in WSN}

To determinate the efficiency and lifetime of network in sensor network, there are several factors that should be consider like total number of sensor nodes, node arrangement as well as node density[23] which further useful in to determine the performance metric like network lifetime, throughput, energy consumption .Figure 2 characterizes the performance metric of the sensor network.

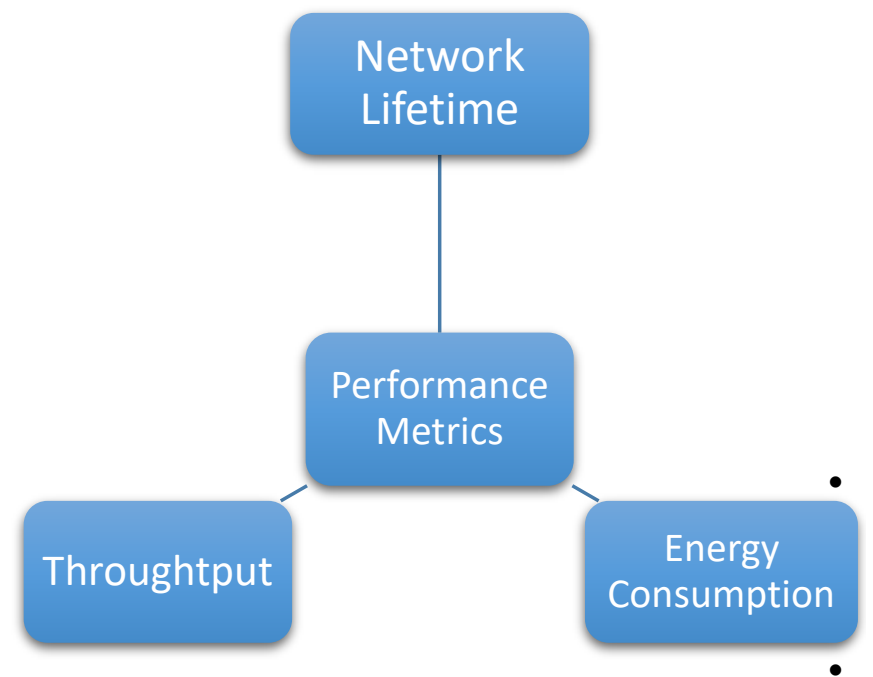

Fig. 2: Performance metrics in WSN

These parameters can be defined as given below.

\section{A. Network lifetime}

There is various definition of system lifetime which mostly depends upon the system necessities. The authors in[24][25] suggested some important point regarding the network lifetime definition which is given below:

- Round as soon as the first number of sensor node expired(FND)

- $\quad$ Round when $\beta$ number of sensor nodes(some nodes) expired out of entire nodes

- Round when last number of node expired(LND)

\section{B. Network throughtput}

Throughput is basically dealings information that a system can practice in a specified time period. In WSN system throughput defines total number of data packets which is communicated to the BS.

\section{Energy consumption}

It is amount of energy disbursed by sensor nodes in system. Figure 3 demonstrates energy Scheme of WSN of total k number of bit which is transmitted over the distance $d$ [9]

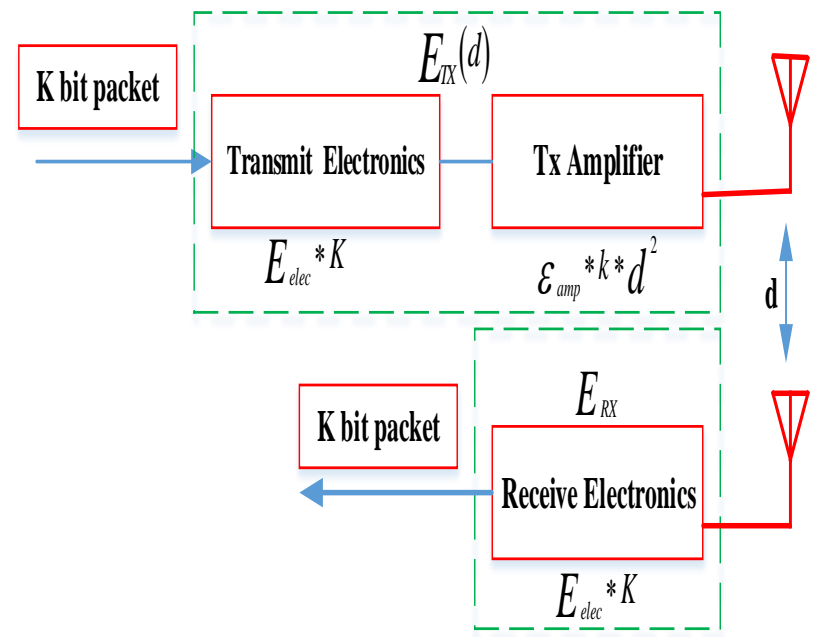

Fig. 3: Energy Scheme plot in WSN

The given below equation (1) and (2) are mainly used for communication and getting energy calculation for total $\mathrm{k}$ number of bit.

$E_{T X}(k, d)= \begin{cases}k E_{\text {elec }}+k \varepsilon_{f s} d^{2} & d<d_{0} \\ k E_{\text {elec }}+k \varepsilon_{\text {amp }} d^{4} & d \geq d_{0}\end{cases}$

$E_{R X}(k)=k E_{\text {elec }}$

Where

$E_{T X}$ is the energy transmission and $E_{R X}$ is energy response of total $\mathrm{k}$ number of bits in the direction of distance $\mathrm{d}$.

$E_{\text {elec }}$ iselectronic energy compulsory for modulation, coding and filtering.

$E_{D A}$ isenergy compulsory for data accumulation.

$\varepsilon_{f s}$ and $\varepsilon_{a m p}$ are the amplification energy.

Equation (3) is basically used to find the $E_{\text {avg }}$ per round which is given as:

$$
\begin{aligned}
& E_{\text {avg }} \\
& =\frac{\text { Total energy consumption of } 1^{\text {st }} \text { node expired }}{\text { Number of rounds before the } 1^{\text {st }} \text { node expired }}
\end{aligned}
$$

\section{Success rate}

Success rate in WSN is stated as ratio of total data packets received by the $\mathrm{BS}$ to the total data packets which are communicated from nodes. 


\section{Varinats of leach protocols}

\subsection{LEACH protocol}

Generally, there are two approach of node deployment. Either node can be deployed randomly or in deterministic way. Deterministic methodology is unrealistic in many situations like military uses as well as in monitoring situation. The haphazard arrangement tactic is more realistic in so many applications[20]. To prolong lifetime of sensor network by enhancing energy effectiveness is foremost agreements nowadays. Network lifetime is totally depend on the life expectancy of battery and necessity for enhancing energy effectiveness which take algorithms into account that have been a leading investigation part in WSN. Indeed, to diminish energy feeding cost triggered by congestion of communiqué amongst all the nodes.

There are basically two types of clustering in the network. One is Homogenous clustering and another one is heterogeneous clustering. As name suggested homogenous means all the nodes in WSN have the equal energy. Furthermore, $\mathrm{CH}$ comprehends data accumulation to wrapping info. Regarding LEACH protocol, it goes through several rounds. In starting, clusters are molded with set-up stage which is tracked by steady state stage[17].

\section{a) Setup phase}

The main work in first phase is to be selection of the node which will become further $\mathrm{CH}$. The selection of $\mathrm{CH}$ is done by using random number which must belongs among 0 and 1 that is equating by aT $(n)$ which is evaluated by equation (4)

$$
T(n)=\left\{\begin{array}{cl}
\frac{P}{1-P(r \bmod 1 / P)} & \text { if } n \in G \\
0 & \text { otherwise }
\end{array}\right.
$$

Where

- $\quad P$ is proportion of choosing $\mathrm{CHs}$.

- $\quad r$ is present round.

- $\quad G$ is group of nodes which have not been $\mathrm{CHs}$ in $1 / P$ rounds After choosing the $\mathrm{CH}$, the $\mathrm{CH}$ initiates communications this info to notify normal node in system for the determination to turn out to be a master node.

\section{b) Steady State phase}

In this phase master nodes every so often collect data and transfer to the $\mathrm{CH}$. Now steady state process is separated into different frames that are further break up in different time slots of same period. Master sensor nodes forward gathered information to the corresponding $\mathrm{CH}$ throughout their assigned communication.

\subsection{KLEACH Protocol}

Figure5 represents the K-means protocols. K-mean technique is basically responsible for determining the centroid in order to decide the cluster formation. Indeed, K-mean technique is on the basis of determination of Euclidian distance method. Subsequently, $\mathrm{CH}$ choice mainly is determined by on residual energy of sensor nodes. Therefore, the dominant node collects info regarding node id, coordinate of node and remaining energy of most of the sensor nodes and collect this info in list of dominant nodes.

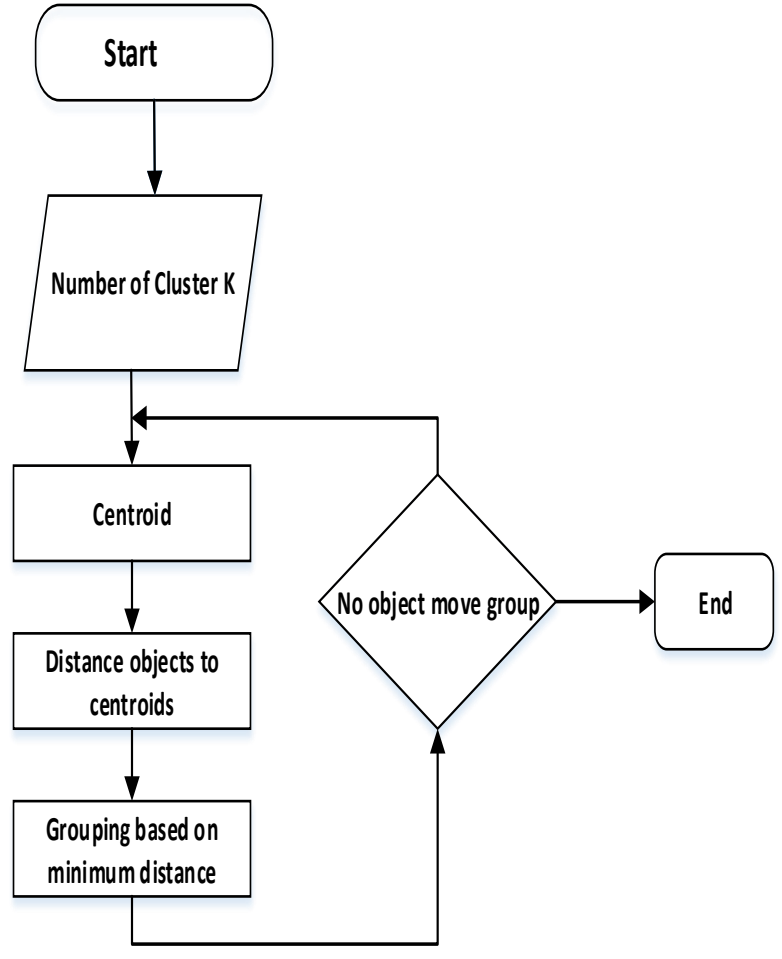

Fig. 4: Flow Process of K-mean Protocol

After getting this info from entirely sensor nodes, accomplishment of clustering is finished[26]. Regarding the LEACH algorithm, it operates through various rounds. In beginning, clusters are molded in set-up stage tracked by steady-state stage as soon as data packets are transmitted from sensor nodes to the $\mathrm{CH}$ and further to BS. However, KLEACH algorithm practices K-means clustering in principal phase. In the subsequent stage, KLEACH accepts the identical action which is similar to steady state phase in LEACH protocol.

\section{Simulation results and analysis}

In this article, MATLAB tool is used to estimate the differentdifferent performance metric in WSN. Simulation Parameter and Simulation Variable are given in Table1 and in Table2 respectively.

Table 1: Simulation parameter and its value

\begin{tabular}{cc}
\hline Parameters & Value \\
\hline $\boldsymbol{E}_{\text {elec }}$ & $40 \mathrm{~nJ} / \mathrm{bit}$ \\
$\boldsymbol{\varepsilon}_{\boldsymbol{f s}}$ & $8 \mathrm{pJ} / \mathrm{bit} / \mathrm{m}^{2}$ \\
$\boldsymbol{\varepsilon}_{\boldsymbol{a m p}}$ & $0.0012 \mathrm{pJ} / \mathrm{bit} / \mathrm{m}^{4}$ \\
$\boldsymbol{E}_{\boldsymbol{D A}}$ & $5 \mathrm{~nJ} / \mathrm{bit}$ \\
$\mathbf{k}$ & $4000 \mathrm{bits}$ \\
\hline
\end{tabular}

Table 2: Simulation Variable and its set up value

\begin{tabular}{lc}
\hline Simulation Variable & Set up values \\
\hline Sensor area & $150 \mathrm{~m} \times 150 \mathrm{~m}$ \\
No. of sensor nodes & 100 to 1000 nodes \\
Initial energy of nodes & $0.5 \mathrm{~J}$ \\
Base Station location & $(50 \mathrm{~m}, 50 \mathrm{~m})$ \\
\hline
\end{tabular}

Some assumptions have been taken while performing the simulation which is given below:

- All sensor nodes have equalpreliminary energy.

- Sensor nodes are not in mobility.

- Packets must be transmitted appropriately by sensor nodes to the $\mathrm{BS}$ and it must be received by BS. 
- Initially BS creates accessible address localization for all sensor nodes.

- Maximum number of $\mathrm{CH}$ should be around $6 \%$ of total available nodes in the network as in.

Initially, performance metric of LEACH as well as KLEACH protocol have been investigated on the foundation of various factors like number of data packets received, sensor nodes lifetime and consumption of energy by nodes in the system. So that we can judge how KLEACH protocol performance has been enhanced compared to LEACH routing protocol. Figure5 reflects the contrast between KLEACH system lifetime and LEACH network lifetime. Graph has been plotted between total numbers of nodes alive versus number of rounds. Based on this plot, one can see that all nodes in LEACH protocol survives up to 1355 rounds only but in case of KLEACH protocol nodes alive till 2607 rounds. Based on this observation we must say that network lifetime of KLEACH protocol has been enhanced compared to the existing LEACH protocol.

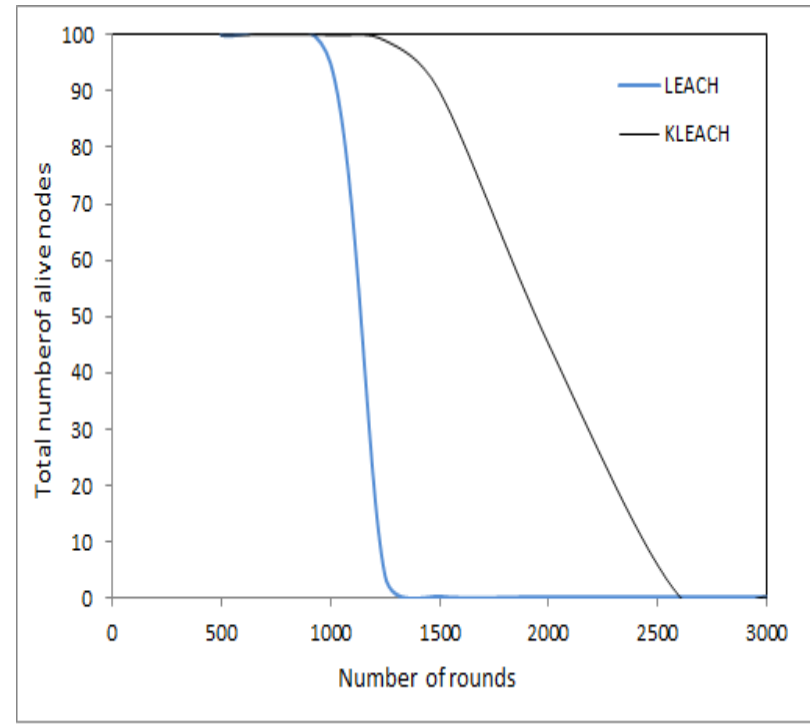

Fig. 5: Network lifetime

Figure 6 reflects the graph of remaining energy of KLEACH as well as LEACH protocol. Graph has been plotted between Residual energy versus number of rounds. We can see that how energy is consumed in nodes in both the protocol. Total preliminary energy of system is taken as $50 \mathrm{~J}$ that gradually falls up to 2200 rounds and debauched energy is realized in 2607 rounds in KLEACH protocol while in case of LEACH protocol energy falls up to 1247 rounds and debauched energy is achieved in 1355 rounds. Hence, it displays the better performance of KLEACH protocol over the LEACH protocol.

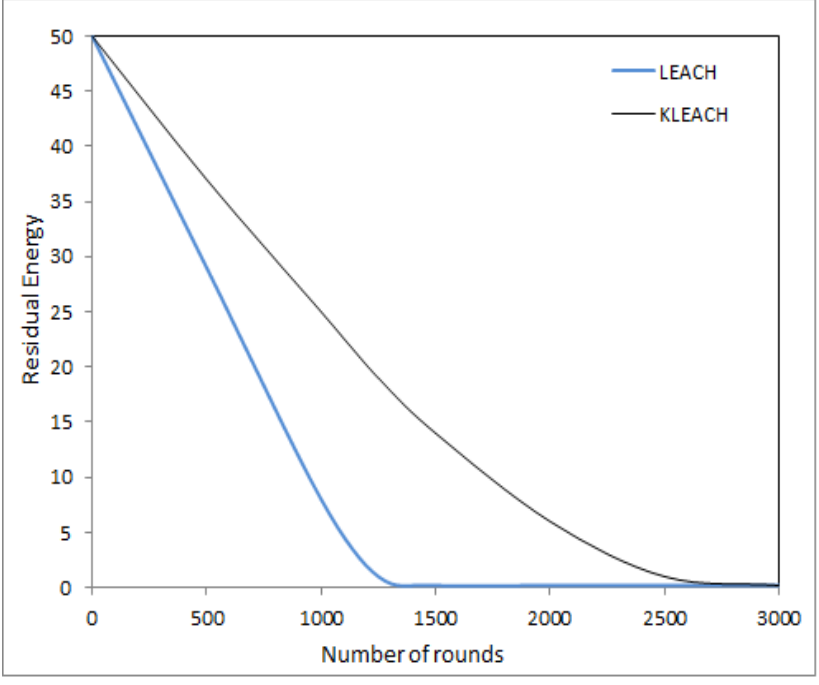

Fig. 6: Residual energy

Figure7 reflects the packets received by BS in both the protocol. Graph has been plotted between total numbers of packet received by BS to the number of rounds. In other word we can say that Figure7 displays throughput progress based on total number of rounds in these cases. It is clearly seen that packet received by BS in case of KLEACH is far better than LEACH protocol. Till 1000 rounds packets received is same in both the protocols but as number of rounds increases packet received is almost constant in case of LEACH protocol but in case of KLEACH protocol it keeps increasing. This statement can also be defensible by circumstance that KLEACH system lifetime is more compared to the LEACH network lifetime.

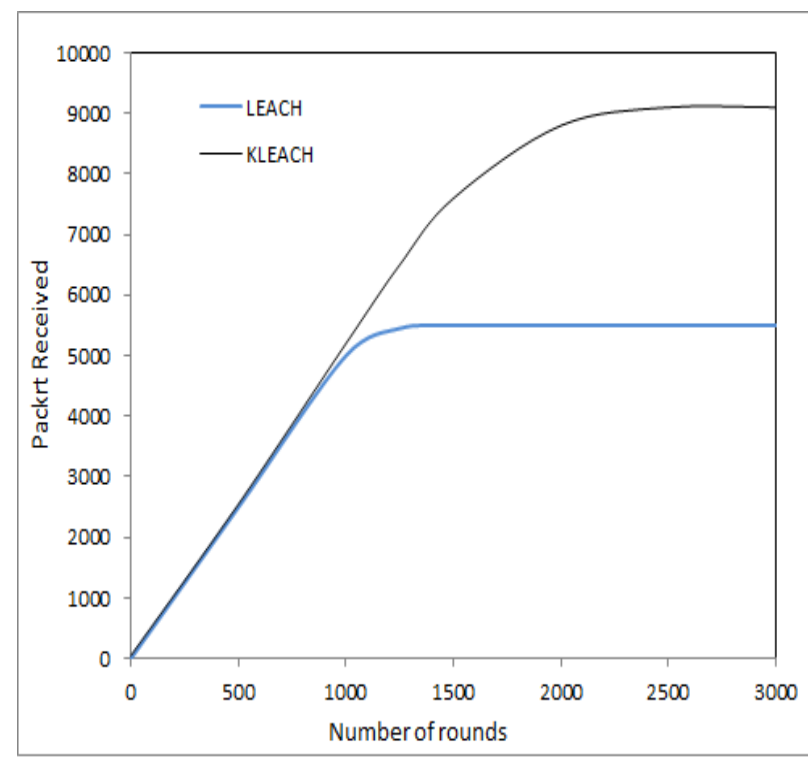

Fig. 7: Total packet received

Throughput progress mainly depends on total number of node that has been taken in each round for LND and FND for both the protocol. It is clear from Figure8 and Figure9 is that total number of packet received by KLEACH protocol is larger than the LEACH Protocol at any instance of time. This statement can also be justified in the sense that network lifetime of KLEACH protocol is more compared to the LEACH protocol. 


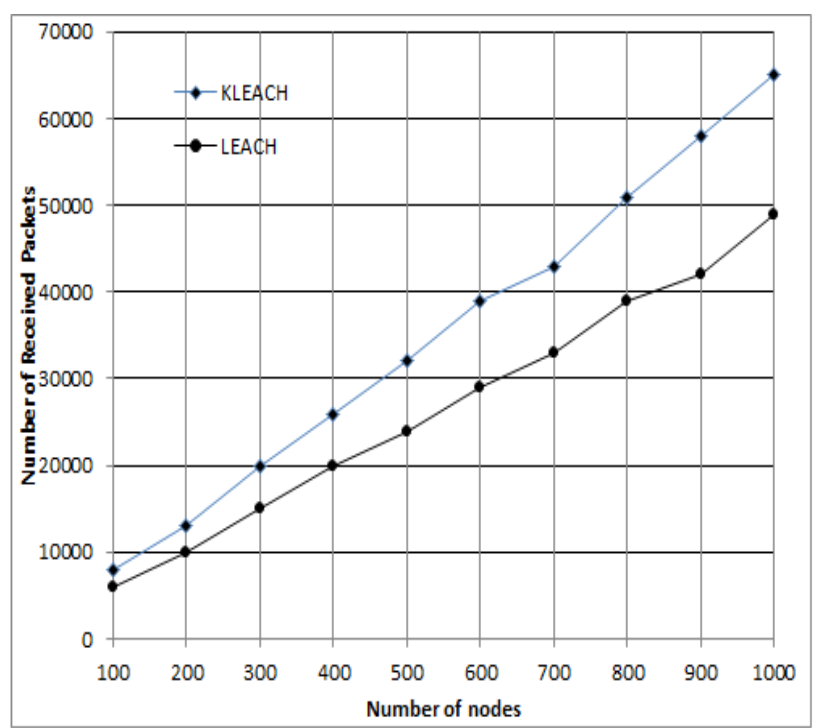

Fig. 8: Total number of packet received in $F N D_{\text {round }}$

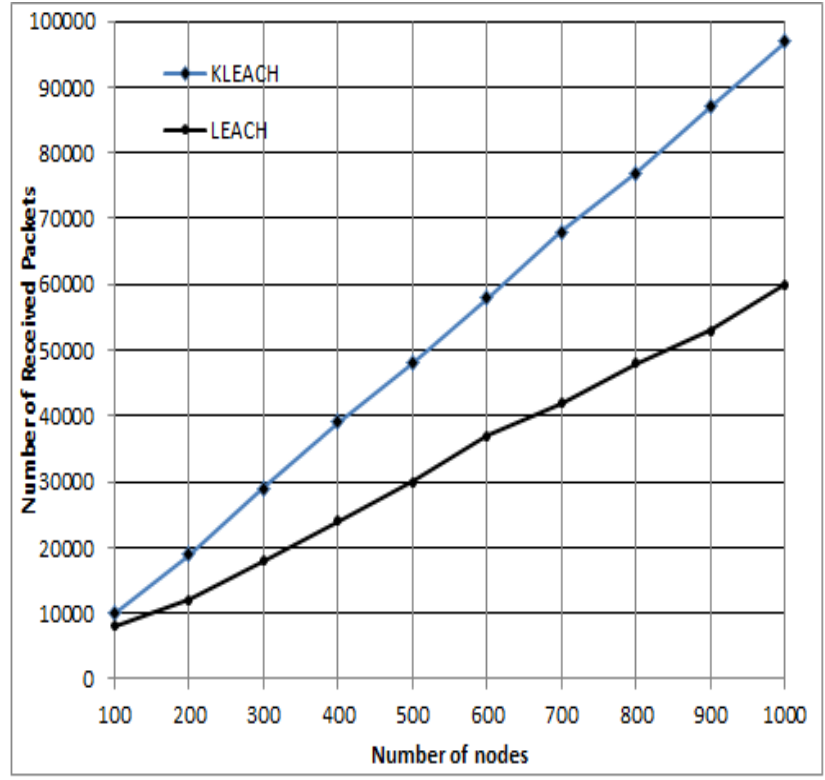

Fig. 9: Total number of packet received in $L N D_{\text {round }}$

In the same way energy consumption of protocol also depends on total number of node that has been taken in each round for LND and FND for both the protocol. It is clear seen from the Figure 10 is that energy feeding is relatively less in KLEACH protocol compared to the LEACH protocol. Also energy saving gain ratio is about $38 \%$ and $30 \%$ in the KLEACH and LEACH protocol respectively. Table 3 demonstrations the performance metrics comparison between KLEACH and LEACH protocol. Network lifetime of KLEACH protocol is almost increased by $40 \%$ and energy consumption is also decrease by almost $38 \%$ which reflects the good agreement of proposed algorithm compared to existing algorithm.

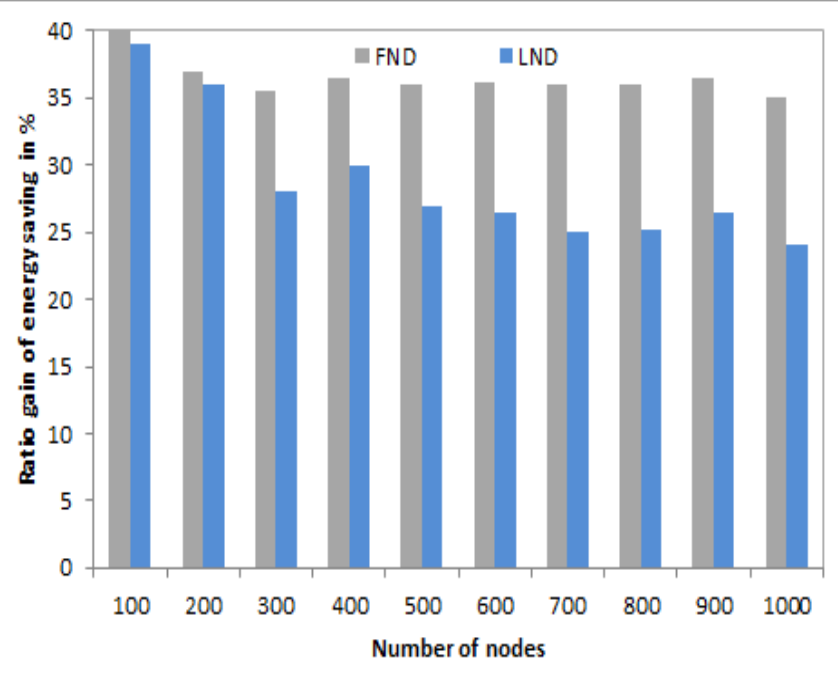

Fig. 10: Gain for energy saving in $F N D_{\text {round }}$ and $L N D_{\text {round }}$

Table 3: Performance Metric Comparison

\begin{tabular}{lc}
\hline Metrics & $\begin{array}{c}\text { Improved Performances Over } \\
\text { LEACH }\end{array}$ \\
\hline Data packets received in FND & Improved by $39.5 \%$ \\
$\begin{array}{l}\text { Average data packets received } \\
\text { ration }\end{array}$ & Incresed by $60 \%$ \\
Network life & Incresed by $40 \%$ \\
Average energy consumption & Decreased by $38 \%$ \\
\hline
\end{tabular}

\section{Conclusion}

In this paper numerous performance factors like network lifetime, energy consumption and data packets received by BS have been performed for different network parameters. The performance of KLEACH and LEACH protocol has been measured to find the network lifetime and how much network is energy efficient. The results display that KLEACH protocol has better network lifetime and energy consumption is also less compared to the existing LEACH protocol. Also total number of packet received by BS in case of KLEACH is more compared to the LEACH over rounds. Furthermore, KLEACH delivers a satisfactory performance metric compared to LEACH protocol. This study can also to extend to examine other parameter like delivery data packets and latency. Moreover, it would be fascinating to study these parameters by means of other clustering methods and make a comparison with outdated routing protocols.

\section{References}

[1] J. Yick, B. Mukherjee, and D. Ghosal, "Wireless sensor network survey," Comput. Networks, vol. 52, no. 12, pp. 2292-2330, 2008.

[2] J. N. Al-Karaki and a. E. Kamal, "Routing Techniques in Wireless Sensor Networks: A Survey," IEEE Wirel. Commun., vol. 11, no. 6, pp. 6-28, 2004.

[3] M. A. Razzaque, C. Bleakley, and S. Dobson, "Compression in wireless sensor networks: A survey and comparative evaluation," ACM Trans. Sens. Networks, vol. 10, no. 1, pp. 5-44, 2013.

[4] C. F. García-hernández, P. H. Ibargüengoytia-gonzález, J. García-hernández, and J. a Pérez-díaz, "Wireless Sensor Networks and Applications : a Survey," J. Comput. Sci., vol. 7, no. 3, pp. 264-273, 2007.

[5] E. Egea-Lopez, J. Vales-Alonso, A. Martinez-Sala, P. PavonMarñio, and J. Garcia-Haro, "Simulation scalability issues in wireless sensor networks," IEEE Communications Magazine, vol. 44, no. 7. pp. 64-73, 2006.

[6] K. Akkaya and M. Younis, "A survey on routing protocols for wireless sensor networks," Ad Hoc Networks, vol. 3, no. 3. pp. 
325-349, 2005.

[7] X. Liu, "A survey on clustering routing protocols in wireless sensor networks," Sensors (Switzerland), vol. 12, no. 8. pp. 11113-11153, 2012.

[8] X. Liu, "A Survey on Clustering Routing Protocols in Wireless Sensor Networks," Sensors, vol. 12, no. 8, pp. 11113-11153, 2012

[9] W. Guo and W. Zhang, "A survey on intelligent routing protocols in wireless sensor networks," Journal of Network and Computer Applications, vol. 38, no. 1. pp. 185-201, 2014.

[10] N. A. Pantazis, S. A. Nikolidakis, and D. D. Vergados, "EnergyEfficient Routing Protocols in Wireless Sensor Networks: A Survey," IEEE Commun. Surv. Tutorials, vol. 15, no. 2, pp. 551591, 2013.

[11] S. A. Nikolidakis, D. Kandris, D. D. Vergados, and C. Douligeris, "Energy efficient routing in wireless sensor networks through balanced clustering," Algorithms, vol. 6, no. 1, pp. 2942, 2013.

[12] S. Ehsan and B. Hamdaoui, "A survey on energy-efficient routing techniques with QoS assurances for wireless multimedia sensor networks," IEEE Communications Surveys and Tutorials, vol. 14, no. 2. pp. 265-278, 2012.

[13] W. B. Heinzelman, A. P. Chandrakasan, and H. Balakrishnan, "An application-specific protocol architecture for wireless microsensor networks," IEEE Trans. Wirel. Commun., vol. 1, no. 4, pp. 660-670, 2002

[14] E. Hossain and K. Leung, Wireless mesh networks: Architectures and protocols. 2007

[15] M. Kohvakka, J. Suhonen, M. Kuorilehto, V. Kaseva, M Hännikäinen, and T. D. Hämäläinen, "Energy-efficient neighbor discovery protocol for mobile wireless sensor networks," Ad Hoc Networks, vol. 7, no. 1, pp. 24-41, 2009.

[16] N. D. Tan and N. D. Viet, "SSTBC: Sleep scheduled and treebased clustering routing protocol for energy-efficient in wireless sensor networks," in Proceedings - 2015 IEEE RIVF International Conference on Computing and Communication Technologies: Research, Innovation, and Vision for Future, IEEE RIVF 2015, 2015, pp. 180-185.

[17] T. M. Rahayu, S. G. Lee, and H. J. Lee, "Survey on LEACHbased security protocols," in International Conference on Advanced Communication Technology, ICACT, 2014, pp. 304 309.

[18] S. K. Singh, P. Kumar, and J. P. Singh, "A Survey on Successors of LEACH Protocol," IEEE Access, vol. 5. pp. 4298-4328, 2017.

[19] Z. Deng and B. Qi, "Three-layered routing protocol for WSN based on LEACH algorithm," Wireless, Mob. Sens. Networks, 2007. (CCWMSN07). IET Conf., pp. 72-75, 2007

[20] S. Tyagi and N. Kumar, "A systematic review on clustering and routing techniques based upon LEACH protocol for wireless sensor networks," Journal of Network and Computer Applications, vol. 36, no. 2. pp. 623-645, 2013.

[21] J. Xu, N. Jin, X. Lou, T. Peng, Q. Zhou, and Y. Chen, "Improvement of LEACH protocol for WSN," in Proceedings 2012 9th International Conference on Fuzzy Systems and Knowledge Discovery, FSKD 2012, 2012, pp. 2174-2177.

[22] F. Xiangning and S. Yulin, "Improvement on LEACH Protocol of Wireless Sensor Network," 2007 Int. Conf. Sens. Technol Appl., vol. 9, no. 2, pp. 260-264, 2007.

[23] L. Alazzawi and A. Elkateeb, "Performance Evaluation of the WSN Routing Protocols Scalability," J. Comput. Syst. Networks, Commun., vol. 2008, pp. 1-9, 2008.

[24] I. Dietrich and F. Dressler, "On the lifetime of wireless sensor networks," ACM Trans. Sens. Networks, vol. 5, no. 1, pp. 1-39, 2009.

[25] Y. Chen and Q. Zhao, "On the lifetime of wireless sensor networks," IEEE Commun. Lett., vol. 9, no. 11, pp. 976-978, 2005.

[26] A. Fahad et al., "A survey of clustering algorithms for big data: Taxonomy and empirical analysis," IEEE Trans. Emerg. Top. Comput., vol. 2, no. 3, pp. 267-279, 2014.

[27] Rajesh, M., and J. M. Gnanasekar. \&quot;Congestion control in heterogeneous wireless ad hoc network using FRCC.\&quot Australian Journal of Basic and Applied Sciences 9.7 (2015) 698-702.

[28] S.V.Manikanthan and V.Rama“Optimal Performance Of Key Predistribution Protocol In Wireless Sensor Networks" International Innovative Research Journal of Engineering and
Technology ,ISSN NO: 2456-1983,Vol-2,Issue -Special -March 2017.

[29] T. Padmapriya and V. Saminadan, "Inter-cell Load Balancing Technique for Multi- class Traffic in MIMO - LTE - A Networks", International Conference on Advanced Computer Science and Information Technology, Singapore, vol.3, no.8, July 2015. 